\title{
Caracterización de las canales ovinas producidas en México
}

\section{Characterization of sheep carcasses produced in Mexico}

\author{
José Armando Partida de la Peña* Francisco Gerardo Ríos Rincón ${ }^{\mathrm{b}}$, Lino de la Cruz Colín, Ignacio Arturo \\ Domínguez Varad, Germán Buendía Rodríguez ${ }^{\mathrm{a}}$
}

\section{RESUMEN}

Se caracterizaron 1,000 canales ovinas producidas en México. Los datos se procesaron a través de estadística descriptiva y mediante un diseño totalmente al azar. Del total de canales evaluadas $82.2 \%$ correspondieron a machos y $17.8 \%$ hembras. Se identificaron 53 genotipos (11 razas puras y 42 cruzamientos diversos). El peso al faenado promedió $43.2 \pm 7.2 \mathrm{~kg}(20.4$ a 85.7), el peso medio de la canal fría fue $22.0 \pm 4.1 \mathrm{~kg}(11.3$ a 50.0$)$ y tuvo un rendimiento medio de 50.0 $5.1 \%\left(34.7\right.$ a 73.5), el área del ojo de chuleta promedió $14.8 \pm 3.8 \mathrm{~cm}^{2}(2.7$ a 29.7$)$, el espesor de la grasa subcutánea promedió $3.1 \pm 1.6 \mathrm{~mm}(1.0$ a 10.0) y el pH $5.5 \pm 0.2(5.0$ a 6.5$)$. El sistema de producción intensiva mostró mayores valores en peso a la matanza, peso de la canal fría y área del ojo de chuleta $\left(44.4 \pm 5.9 \mathrm{~kg}, 22.8 \pm 3.3 \mathrm{~kg}\right.$ y $15.5 \pm 3.6 \mathrm{~cm}^{2}$, respectivamente) que el semiintensivo (43.6 $\pm 5.3 \mathrm{~kg}, 19.9 \pm 3.1 \mathrm{~kg}$ y $\left.13.8 \pm 2.5 \mathrm{~cm}^{2}\right)$ y el extensivo $\left(36.4 \pm 9.7,19.6 \pm 2.6 \mathrm{~kg}\right.$ y $\left.9.6 \pm 2.6 \mathrm{~cm}^{2}\right)$. Los machos presentaron mayores valores que las hembras en peso al sacrificio (44.3 $\pm 6.7 \mathrm{vs} 40.1 \pm 6.1 \mathrm{~kg})$, peso de la canal fría (22.2 $\pm 3.8 \mathrm{vs} 21.2 \pm 4.4 \mathrm{~kg})$ y área del ojo de chuleta $\left(15.2 \pm 3.7\right.$ vs $\left.12.7 \pm 3.7 \mathrm{~cm}^{2}\right)$. La gran diversidad en el fenotipo y condición de los ovinos para abasto originó una amplia variabilidad en las propiedades de la canal, que limita el mercado formal de la carne ovina, por lo que convendría reordenar la producción ovina nacional dirigiéndola hacia una condición de calidad más uniforme.

PALABRAS CLAVE: Caracterización, Ovinos, Calidad, Canal.

\section{ABSTRACT}

One thousand $(1,000)$ sheep carcasses produced in Mexico were characterized. Data were processed through descriptive statistics and using a totally random design. From total carcasses, $82.2 \%$ were males and $17.8 \%$ females. Fifty three (53) genotypes (11 pure breeds and 42 different crosses) were identified. Slaughter weight averaged 43.2 $\pm 7.2 \mathrm{~kg}(20.4$ to 85.7$)$, cold carcass weight averaged $22.0 \pm 4.1 \mathrm{~kg}(11.3$ to 50.0$)$ carcass yield was $50.0 \pm 5.1 \%$ (34.7 to 73.5), rib eye area averaged $14.8 \pm 3.8 \mathrm{~cm}^{2}(2.7$ to 29.7$)$, subcutaneous fat thickness had a mean of $3.1 \pm$ $1.6 \mathrm{~mm}(1.0$ to 10.0) and pH averaged $5.5 \pm 0.2(5.0$ to 6.5). The intensive production system showed higher values in slaughter weight, cold carcass weight and rib eye area $\left(44.4 \pm 5.9 \mathrm{~kg}, 22.8 \pm 3.3 \mathrm{~kg}\right.$ and $15.5 \pm 3.6 \mathrm{~cm}^{2}$, respectively) than the semi-intensive system $\left(43.6 \pm 5.3 \mathrm{~kg}, 19.9 \pm 3.1 \mathrm{~kg}\right.$ and $\left.13.8 \pm 2.5 \mathrm{~cm}^{2}\right)$ and extensive system $\left(36.4 \pm 9.7,19.6 \pm 2.6 \mathrm{~kg}\right.$ and $\left.9.6 \pm 2.6 \mathrm{~cm}^{2}\right)$. Males had higher values than females in slaughter weight $(44.3 \pm 6.7$ $v s 40.1 \pm 6.1 \mathrm{~kg})$, cold carcass weight $(22.2 \pm 3.8 \mathrm{vs} 21.2 \pm 4.4 \mathrm{~kg})$ and rib eye area $\left(15.2 \pm 3.7 \mathrm{vs} 12.7 \pm 3.7 \mathrm{~cm}^{2}\right)$. The great diversity in phenotypes and different lamb conditions caused considerable variability in carcass traits, which limits the formal lamb market, so sheep domestic production should be reordered towards a more uniform quality condition.

KEY WORDS: Characterization, Sheep, Quality, Carcass.

\footnotetext{
Recibido el 21 de junio de 2016. Aceptado el 23 de octubre de 2016.

${ }^{a}$ Centro Nacional de Investigación Disciplinaria en Fisiología y Mejoramiento Animal, INIFAP. México.

${ }^{\mathrm{b}}$ Facultad de Medicina Veterinaria y Zootecnia, Universidad Autónoma de Sinaloa. México.

c Sitio Experimental Hidalgo, INIFAP. México.

${ }^{d}$ Facultad de Medicina Veterinaria y Zootecnia, Universidad Autónoma del Estado de México. México.

* Autor de correspondencia: partida.jose@inifap.gob.mx.
} 


\section{INTRODUCCIÓN}

Generalmente, la carne de borrego es un producto de bajo consumo en México, pues durante los últimos 25 años el consumo nacional aparente ha variado entre 500 y $950 \mathrm{~g} / \mathrm{año} / \mathrm{per}$ cápita $^{(1)}$. Esa moderada participación de la carne ovina en la canasta alimenticia de los mexicanos se explica por varios factores, entre los que sobresalen los siguientes: su costo es elevado con respecto a otras carnes (pollo y cerdo), el $90 \%$ del consumo de carne de borrego se basa en un solo platillo típico (barbacoa) que se come en forma ocasional, la oferta de carne es cíclica a través del año (con una mayor demanda durante el último trimestre) y la importación (de carne congelada o animales en pie) está sujeta a la paridad existente entre el peso y el dólar. No obstante todo lo anterior, se estima que para tener una disponibilidad limitada a sólo 750 g/persona en el año 2016, además de la producción nacional $(\sim 60,000 \mathrm{t})^{(2,3,4)}$, se requerirán importar más de 30,000 t de carne en canal, que significan la salida de divisas por una cantidad superior a los 3,000 millones de pesos.

Para el desarrollo de la ovinocultura, México posee una orografía muy diversa, con una climatología que cambia fuertemente de un lugar a otro y con múltiples recursos naturales que son aprovechados en distintos sistemas de producción, que difieren por su modalidad (estabulación, semiestabulación y pastoreo), por su grado de intensidad (intensivo, semiintensivo, extensivo) y por el nivel tecnológico que tienen (tecnificado, semitecnificado y tradicional). Esta amplia gama en los sistemas productivos origina fluctuaciones estacionales en la disponibilidad de ganado para el abasto, y ocasiona mucha irregularidad en el tipo y la condición de los animales que se producen, lo que se ve reflejado en la calidad del producto final. Esta situación tiene una fuerte discrepancia con los requerimientos del mercado que demandan regularidad, calidad y uniformidad, y coloca a los productores mexicanos en una posición totalmente desventajosa frente a la competencia internacional, la cual cada vez es más intensa y dinámica ${ }^{(5)}$. Por todo lo anterior, el objetivo del presente trabajo fue caracterizar las propiedades de las canales ovinas que se producen en el país para identificar la situación de la oferta actual y contribuir con información que ayude a orientar la producción hacia una condición que permita satisfacer más adecuadamente las demandas y preferencias de los mercados.

\section{MATERIAL Y MÉTODOS}

El trabajo se realizó en el Laboratorio de Carnes del Centro Nacional de Investigación Disciplinaria en Fisiología y Mejoramiento Animal del INIFAP, ubicado en Ajuchitlán, Qro.

Para el muestreo de canales se empleó el método intencional 0 de conveniencia ${ }^{(6)}$. El muestreo se llevó a cabo en 14 de las entidades federativas con la mayor producción ovina del país (Estado de México, Hidalgo, Veracruz, Zacatecas, Puebla, Jalisco, Coahuila, Sinaloa, San Luis Potosí, Guanajuato, Querétaro, Tabasco, Chiapas y Yucatán) e incluyó la evaluación de 1,000 canales de los tres principales sistemas productivos (intensivo, semiintensivo y extensivo), en unidades de producción (UP) que autorizaron el acceso a sus instalaciones, brindaron información sobre la productividad primaria antes de la matanza (sexo, raza, peso, tipo de alimentación, sistema de producción, etc.) y permitieron realizar la evaluación de las canales.

\section{Evaluación de la canal}

Todos los ovinos se pesaron al salir de las UP, se mantuvieron en corrales de espera, en los que no se les proporcionó alimento durante un periodo de $12 \pm 2 \mathrm{~h}$ (únicamente se les dio agua de bebida), y se sacrificaron siguiendo los procedimientos comerciales establecidos en cada uno de los rastros o los lugares de matanza. Después del sacrificio se pesaron las canales calientes, se dejaron orear durante $4 \mathrm{~h}$ y después se introdujeron en una cámara frigorífica $\left(2 \pm 2{ }^{\circ} \mathrm{C}\right)$ durante $24 \mathrm{~h}$. Pasado este tiempo, las canales se pesaron nuevamente para registrar su peso frío, del cual se obtuvo el rendimiento comercial en frío ${ }^{(7)}$. Se midió el $\mathrm{pH}$ final con un potenciómetro provisto con electrodo de penetración y termómetro (Hanna ${ }^{\circledR}$ HI-99163 Woonsocket RI USA). Posteriormente, se realizaron las mediciones morfométricas de las canales 
(longitud de la canal y de la pierna, perímetro y ancho de la grupa, ancho mayor y menor del tórax, perímetro torácico y profundidad interna del tórax) de acuerdo con la metodología descrita por Ruiz de Huidobro(8); además, se calculó el índice de compacidad de la canal [peso $(\mathrm{kg})$ / longitud total $(\mathrm{cm})$ ]. Las mediciones de longitud se efectuaron con una cinta métrica, y para la anchura se empleó el "bastón de Aparicio".

La clasificación de las canales se llevó a cabo de acuerdo con la norma mexicana NMX-FF-106-SCFI2006. Para medir las dimensiones del músculo Longissimus dorsi se cuarteó la media canal

Cuadro 1. Principales tipos genéticos ovinos observados en el muestreo

\begin{tabular}{lc}
\hline Genotipo & $\%$ \\
\hline Razas puras: & \\
Katahdin & 11.0 \\
Pelibuey & 5.1 \\
Rambouillet & 5.0 \\
Suffolk & 3.4 \\
Dorper & 2.8 \\
Black Belly & 1.7 \\
Charollais & 1.3 \\
Texel & 1.3 \\
Ile de France & 0.2 \\
Dorset & 0.5 \\
Hampshire & $\underline{0.5}$ \\
Subtotal & 32.8 \\
Cruzamientos: & \\
Pelibuey x Katahdin & 16.3 \\
Pelibuey x Dorper & 9.6 \\
Katahdin x Suffolk & 100.0 \\
Katahdin x Charollais & 9.0 \\
Katahdin x Dorper & 5.8 \\
Criollo x Katahdin & 5.4 \\
Pelibuey x Dorset & 2.5 \\
Pelibuey x Black Belly & 2.5 \\
Katahdin x Texel & 2.2 \\
Pelibuey x Criollo & 2.1 \\
Otras cruzas & 1.6 \\
\hline Subtotal & 10.2 \\
\hline & 67.2 \\
\hline
\end{tabular}

izquierda sobre el espacio intercostal que se encuentra ubicado entre la $12 .^{a}$ y $13 .^{a}$ vértebras torácicas, utilizando un rotulador de punta fina se dibujó el contorno del músculo en papel acetato y posteriormente se midió la superficie muscular con un planímetro digital (Planix 6, Tamaya Technics Inc. Tokio, Japón). Adicionalmente, con un calibrador digital (Vernier) IP67 (Mitutoyo Inc. Chicago, Illinois, EUA), se midió la distancia del diámetro mayor en sentido medio-lateral y del diámetro menor en sentido dorso-ventral. La medición del espesor de grasa subcutánea también se hizo con el vernier entre la $12 .^{a}$ y $13 .^{a}$ costilla a $4 \mathrm{~cm}$ de la línea media dorsal.

\section{Análisis de los datos}

Los resultados generales se procesaron mediante estadística descriptiva, los datos desagregados (por sistema de producción y sexo) se analizaron por medio de un diseño totalmente al azar, y las medias se compararon con la prueba de Tukey ${ }^{(9)}$ empleando el procedimiento GLM del paquete estadístico SAS ${ }^{(10)}$.

\section{RESULTADOS y DISCUSIÓN}

Las canales evaluadas correspondieron a machos enteros $(82.2 \%)$ y hembras $(17.8 \%)$. En total se identificaron 53 genotipos, de los cuales 11 fueron razas puras y 42 cruzamientos diversos (Cuadro 1); se observa que las razas de pelo sobresalieron, tanto en forma pura como en los cruzamientos, siendo Katahdin y Pelibuey los genotipos más numerosos. Esta elevada participación de los animales de pelo en la genética ovina del país, coincide con los reportes de la Unión Nacional de Ovinocultores ${ }^{(11)} y$, en gran parte, se debe a su alta capacidad de adaptación a diferentes condiciones ambientales ${ }^{(12,13)}$, a la mayor resistencia que presentan estos tipos genéticos a diversas afecciones parasitarias ${ }^{(14,15)}$, y a sus ventajas reproductivas (precocidad, prolificidad y baja presencia de anestro estacional) ${ }^{(16,17)}$.

En el Cuadro 2 se muestran los tipos genéticos con mayor presencia en cada una de las tres principales regiones productoras del país. En la región norte todavía prevalece la raza Rambouillet, 
Cuadro 2. Preponderancia de tipos genéticos ovinos en las tres regiones productoras de México

\begin{tabular}{lll}
\hline & \multicolumn{1}{c}{ Regiones } \\
\hline Norte & Centro & Sur \\
\hline Rambouillet & Suffolk & Pelibuey \\
Pelibuey & Hampshire & Black Belly \\
Dorper & Dorset & Criollo \\
Katahdin & Pelibuey & Katahdin \\
Cruzas de pelo x lana & Katahdin & Dorper \\
& Dorper & Cruzas de pelo \\
& Charollais & \\
& Texel & \\
& lle de France & \\
& East Friesian & \\
& Criollo & \\
& Cruzas de pelo x lana & \\
\hline
\end{tabular}

Realizado con información de la Unión Nacional de Ovinocultores (UNO, 2016).

que fue introducida a finales del siglo XIX en los sistemas extensivos de zonas áridas para producción de lana, pero también se pueden identificar razas de pelo más recientemente introducidas. En la región centro prevalecen las razas Suffolk, Hampshire y Dorset, principalmente en los estados de México, Hidalgo, Puebla y Tlaxcala, pero existe la presencia de varias razas de reciente introducción, así como distintas razas de pelo y un gran número de cruzamientos entre estas dos variedades. En la región sur prevalecen las razas de pelo Pelibuey y Black Belly, así como diversos cruzamientos entre razas de pelo con genotipos de reciente introducción como Katahdin y Dorper. Además, se mantienen núcleos de animales criollos que proporcionan lana para prendas artesanales, principalmente en los estados de Chiapas y Oaxaca.

Los resultados derivados del muestreo general revelaron que el peso al faenado promedió $43.2 \pm$ $7.2 \mathrm{~kg}$, con un rango extremadamente amplio que va de 20.4 a $85.7 \mathrm{~kg}$, el peso de la canal presentó una media de $22.0 \pm 4.1 \mathrm{~kg}$ y un rango de $11.3 \mathrm{a}$ $50.0 \mathrm{~kg}$, el rendimiento en canal fría promedió 50.0 $\pm 5.1 \%$, con un rango de 34.7 a $73.5 \%$.

La variación tan grande que se observó en los genotipos y los diferentes pesos al momento del sacrifico repercutieron en todos los demás parámetros de la canal (morfometría, clasificación y dimensiones del músculo Longissimus dorsi) en los que también se presentaron promedios acordes con la especie ovina, pero rangos muy amplios; por ejemplo, la conformación de la canal promedió 6.1 \pm 1.7 con rango de 2 a 9, el valor medio de la clasificación fue de $1.9 \pm 0.8$, con rango de 1.0 a 4.0, el área del ojo de chuleta promedió $14.8 \pm 3.8$ $\mathrm{cm}^{2}$ con rango de 2.7 a $29.7 \mathrm{~cm}^{2}$, el espesor de la grasa subcutánea tuvo una media de $3.1 \pm 1.6 \mathrm{~mm}$, con valores extremos de 1.0 a $10.0 \mathrm{~mm}$ y el pH promedió $5.5 \pm 0.2$, con un rango que va de 5.0 hasta 6.5.

En términos generales, el sistema de producción intensivo presentó mayores valores en el peso a la matanza, peso de la canal fría y área del ojo de chuleta $(44.4 \pm 5.9 \mathrm{~kg}, 22.8 \pm 3.3 \mathrm{~kg}$ y 15.5 $\pm 3.6 \mathrm{~cm}^{2}$, respectivamente) que el sistema semiintensivo $(43.6 \pm 5.3 \mathrm{~kg}, 19.9 \pm 3.1 \mathrm{~kg}$ y $13.8 \pm 2.5$ $\left.\mathrm{cm}^{2}\right)$ y que el extensivo $(36.4 \pm 9.7 ; 19.6 \pm 2.6 \mathrm{~kg}$ y $9.6 \pm 2.6 \mathrm{~cm}^{2}$ ). En cuanto al efecto del sexo, los machos presentaron valores mayores que las hembras en peso al sacrificio ( $44.3 \pm 6.7$ vs $40.1 \pm$ $6.1 \mathrm{~kg})$, peso de la canal fría $(22.2 \pm 3.8$ vs $21.2 \pm$ $4.4 \mathrm{~kg})$ y áreas del ojo de chuleta $(15.2 \pm 3.7 \mathrm{vs}$ $\left.12.7 \pm 3.7 \mathrm{~cm}^{2}\right)$. Gran parte de la variación que se registró en los parámetros de calidad en la canal, se puede atribuir a dos factores: uno las diferencias en los ritmos de crecimiento originados por el nivel nutricional de los sistemas de producción; y el otro, 
debido a la amplia diversidad genética (diferente grado de precocidad entre razas), que se presenta en las UP del país, y que ha sido ocasionada por la importación de varias razas exóticas, las cuales se han introducido a México con el fin de "mejorar la genética nacional", pero que en muchos casos, se emplean en cruzamientos descontrolados que originan rebaños comerciales con mezclas indefinidas. La literatura internacional es muy escaza en cuanto a la caracterización de la canal ovina, ya que los países industrializados cuentan con sistemas obligatorios de clasificación de canales y tienen una trazabilidad muy precisa, mientras que los países subdesarrollados o en vías de desarrollo únicamente reportan trabajos sobre la caracterización de sus sistemas de producción ovina, ya que la comercialización de los animales se realiza en pie.

Al desagregar los datos por sistema de producción y sexo, se advierten diferencias $(P<0.05)$ en distintas variables. En el Cuadro 3 se muestran algunas características de la canal, observándose que el peso a la matanza fue ( $7.4 \%$ en promedio) mayor en los machos que en las hembras de los tres sistemas de producción, no hubo diferencia
( $P>0.05$ ) entre el sistema intensivo y semiintensivo, pero sí en estos dos sistemas con el extensivo. Estas diferencias en los pesos de matanza son consecuencia de los distintos tipos de animales incluidos, pues en el muestreo se consideraron machos y hembras de diferente genotipo, edad, condición corporal, régimen de alimentación, etc.

A su vez, la variación en los distintos pesos de matanza causó diferencias en los demás parámetros de la canal; por ejemplo, el peso de ésta mostró diferencias $(P<0.05)$ entre sexos en los sistemas extensivo y semiintensivo, pero no en el intensivo. El rendimiento de la canal fría también registró variaciones $(P<0.05)$ entre sexos y entre sistemas de producción, pero no se observó una tendencia clara en esta variable, pues el mayor rendimiento lo obtuvieron los ovinos del sistema extensivo, de quienes se podría esperar que presentaran el rendimiento más bajo debido a que por lo general, son hembras de desecho que con frecuencia llegan gestantes a los centros de procesamiento ${ }^{(18)}$. No obstante, esta variación en el peso y rendimiento de la canal se debe a un gran número de factores intrínsecos (del animal) y extrínsecos (de su

Cuadro 3. Particularidades de canales ovinas (media $\pm D E$ ) procedentes de machos y hembras de tres sistemas de producción en México

\begin{tabular}{|c|c|c|c|c|c|c|}
\hline \multirow{3}{*}{ Variable } & \multicolumn{6}{|c|}{ Sistema de producción } \\
\hline & \multicolumn{2}{|c|}{ Extensivo } & \multicolumn{2}{|c|}{ Semiintensivo } & \multicolumn{2}{|c|}{ Intensivo } \\
\hline & Hembras & Machos & Hembras & Machos & Hembras & Machos \\
\hline Peso de matanza, kg & $34.8 \pm 9.7^{d}$ & $37.8 \pm 7.7^{c}$ & $42.1 \pm 4.7^{b}$ & $43.9 \pm 6.6^{a}$ & $41.0 \pm 5.0^{b}$ & $44.9 \pm 5.8 \mathrm{a}$ \\
\hline Peso canal fría, kg & $19.0 \pm 5.7^{c}$ & $20.1 \pm 5.7^{b}$ & $17.4 \pm 1.4 \mathrm{~d}$ & $20.6 \pm 3.1 b$ & $22.6 \pm 3.3^{a}$ & $22.8 \pm 3.3^{a}$ \\
\hline $\mathrm{R}^{\star}$. Canal fría, \% & $54.1 \pm 6.7$ a & $53.0 \pm 7.0 \mathrm{a}$ & $42.1 \pm 4.6 \mathrm{~d}$ & $47.0 \pm 4.5^{c}$ & $55.1 \pm 4.1 \mathrm{a}$ & $50.9 \pm 4.3 b$ \\
\hline Conformación & $3.3 \pm 0.9 c$ & $3.5 \pm 0.9 c$ & $4.6 \pm 0.5^{b}$ & $6.4 \pm 1.2^{\mathrm{a}}$ & $5.2 \pm 1.4^{b}$ & $6.6 \pm 1.4$ a \\
\hline Clasificación & $3.2 \pm 0.5$ & $3.0 \pm 0.6$ & $2.0 \pm 0.0$ & $1.8 \pm 0.5$ & $2.1 \pm 0.7$ & $1.7 \pm 0.7$ \\
\hline $\mathrm{pH}(24 \mathrm{~h})$ & $5.3 \pm 0.2$ & $5.4 \pm 0.2$ & $5.6 \pm 0.2$ & $5.5 \pm 0.2$ & $5.5 \pm 0.2$ & $5.5 \pm 0.2$ \\
\hline $\mathrm{EGS}, \mathrm{mm}$ & $2.7 \pm 1.0$ & $2.4 \pm 1.7$ & $3.5 \pm 1.0$ & $3.0 \pm 1.2$ & $3.1 \pm 1.0$ & $3.2 \pm 1.6$ \\
\hline \multicolumn{7}{|c|}{ Dimensiones del músculo Longissimus dorsi: } \\
\hline Área, $\mathrm{cm}^{2}$ & $9.0 \pm 2.2 \mathrm{~d}$ & $10.3 \pm 2.3^{c}$ & $12.0 \pm 1.8^{b}$ & $14.3 \pm 2.6^{a}$ & $14.9 \pm 2.9 \mathrm{a}$ & $15.6 \pm 3.6^{a}$ \\
\hline Diámetro mayor, cm & $4.0 \pm 0.1^{c}$ & $4.0 \pm 0.0^{c}$ & $5.4 \pm 0.3^{b}$ & $5.5 \pm 0.5^{b}$ & $6.4 \pm 0.4 \mathrm{a}$ & $5.5 \pm 1.3^{b}$ \\
\hline Diámetro menor, $\mathrm{cm}$ & $2.8 \pm 0.3^{c}$ & $2.8 \pm 0.0^{c}$ & $2.8 \pm 0.3^{c}$ & $3.0 \pm 0.5^{b}$ & $3.5 \pm 0.4 \mathrm{a}$ & $3.4 \pm 0.8^{a}$ \\
\hline \multicolumn{7}{|c|}{$\mathrm{R}^{\star}=$ Rendimiento, $E G S=$ espesor de la grasa subcutánea sobre el músculo $L$. dorsi. } \\
\hline \multicolumn{7}{|c|}{ Clasificación de acuerdo con la Norma NMX-FF-106-SCFI-2006. } \\
\hline \multicolumn{7}{|c|}{ 1= MEX EXT; 2= MEX 1; 3= MEX 2; 4= Fuera de clasificación. } \\
\hline \multicolumn{7}{|c|}{$\begin{array}{l}\text { Conformación: } 9=\text { Excelente }(+), 8=\text { Excelente, } 7=\text { Excelente }(-) ; 6=\text { Buena }(+), 5=\text { Buena, } 4=\text { Buena }(-) ; 3=\text { Deficiente }(+), 2=\text { Deficiente y } 1=\text { Deficiente }(-) \text {. } \\
\text { abcd Letras distintas en el mismo narámetro indican diferencia }(P<0.05) \text {. }\end{array}$} \\
\hline
\end{tabular}


entorno). Dentro de los primeros ocupan un lugar preponderante el peso a la matanza, el estado de madurez, el sexo y la edad de los ovinos, ya que cuanto mayor es el peso de sacrificio mayor es el rendimiento en canal(18-21), porque los ovinos alcanzan un porcentaje más alto de su peso maduro y logran un mejor finalizado(22), pero a peso constante las hembras tienen mayor rendimiento que los machos ${ }^{(14,20,23)}$; asimismo, en ovinos de más edad el tubo digestivo representa un porcentaje mayor con respecto a su peso corporal(21). Por su parte, los factores extrínsecos están más relacionados con el transporte, la duración del tiempo de espera previo al faenado y el tipo de alimentación que reciben durante el periodo de finalización, pues la duración y condiciones del transporte, así como el tiempo previo al sacrificio ocasionan que los ovinos pierdan peso por la movilización de reservas que origina la actividad física y el estrés ${ }^{(24,25,26)}$, y porque eliminan orina y excremento que modifican el peso vivo vacío y el rendimiento de la canal ${ }^{(27,28)}$.

En general, la conformación de la canal fue mejor en los machos que en las hembras, y en cuanto al sistema de producción, sobrepasaron el intensivo y semiintensivo al extensivo, pero no se observaron diferencias $(P>0.05)$ en la clasificación de la canal, promediando $2.3 \pm 0.4$ puntos, que corresponden a una clasificación "MEX 1". Una posible explicación acerca de las diferencias en la conformación y clasificación de la canal entre sistemas de producción es el manejo alimenticio superior que reciben los ovinos en los sistemas intensivo y semiintensivo, que promueve una finalización más completa del animal con mejores proporciones corporales, ya que los resultados de trabajos previos mostraron que al aumentar los niveles de energía en la dieta aumenta el rendimiento en canal(29), se mejora la relación músculo:grasa ${ }^{(30,31)}$, se incrementa el depósito de grasa visceral y ésta se hace más insaturada ${ }^{(32,33)}$. Asimismo, la suplementación alimenticia que se proporciona en los sistemas semiintensivos, aumenta el rendimiento, mejora las características de la canal( ${ }^{(34,35)}$ e incluso mejora el sabor de la carne ${ }^{(36)}$.

No se observaron diferencias $(P>0.05)$ entre sistemas de producción o entre sexos en el pH final
(24 h) de la carne, ni en el espesor de la grasa subcutánea, con promedios de $5.5 \pm 0.2$ y $2.9 \pm 0.9$ $\mathrm{mm}$, respectivamente. Un pH final de 5.5 en la carne ovina se considera totalmente apropiado en ovinos $^{(37)}$, pues al parecer esta especie, en comparación con otras, presenta una menor susceptibilidad al estrés que se origina antes y durante el proceso de matanza(38). En cuanto al engrasamiento de la canal, valores de grasa subcutánea hasta de $3 \mathrm{~mm}$ en corderos livianos o hasta de $6 \mathrm{~mm}$ en corderos pesados, son considerados adecuados y no reciben penalización en la clasificación mexicana de la canal (NMX-FF106-SCFI) ${ }^{(39)}$.

En términos generales, las dimensiones del músculo Longissimus dorsi, fueron menores $(P<0.05)$ en el sistema extensivo que en los otros dos sistemas, lo que coincide con otros investigadores que observaron diferencias entre sistemas de producción ${ }^{(40,41,42)}$, pero el área del músculo $L$. dorsi de los ovinos machos fue mayor (+19.2\%) que el de las hembras sólo en el sistema semiintensivo, siendo similares en ambos sexos en los otros dos sistemas, lo que coincide con varios autores, quienes asocian más las dimensiones del músculo $L$. dorsicon el peso del ovino en vivo(20,36,43). La morfometría de la canal (Cuadro 4) también reveló diferencias $(P<0.05)$ en varios parámetros como la longitud de la canal y de la pierna, el perímetro de la grupa y del tórax, así como en el índice de compacidad de la canal, siendo estos valores mayores en los sistemas intensivo y semiintensivo que en el extensivo. Estas diferencias pueden ser debidas al genotipo del ovino(43-46), pero más específicamente debido a su talla ${ }^{(47)}$ y peso al momento de la matanza ${ }^{(19,20)}$, pues evidentemente las razas de talla grande muestran valores mayores en la morfometría de la canal que las razas pequeñas; asimismo, cuando es mayor el peso al faenado se incrementa $(P<0.05)$ el rendimiento en canal $^{(19,20,43)}$. Por esto, sería recomendable que en la producción de carne ovina se emplearan razas cárnicas especializadas o cruzamientos entre razas de pelo y lana, ya que en trabajos previos en los que se evaluaron más de 20 cruzas terminales, los corderos llegaron al peso de sacrificio antes de los 5 meses de edad, y se obtuvieron excelentes características de la canal, con rendimientos 
Cuadro 4. Mediciones morfométricas en canales ovinas (media $\pm \mathrm{DE}$ ) procedentes de tres sistemas de producción en México

\begin{tabular}{|c|c|c|c|c|c|c|}
\hline \multirow{3}{*}{ Variable (cm) } & \multicolumn{6}{|c|}{ Sistema de producción } \\
\hline & \multicolumn{2}{|c|}{ Extensivo } & \multicolumn{2}{|c|}{ Semiintensivo } & \multicolumn{2}{|c|}{ Intensivo } \\
\hline & Hembras & Machos & Hembras & Machos & Hembras & Machos \\
\hline Longitud de canal & $58.70 \pm 3.4 \mathrm{~d}$ & $60.80 \pm 1.9 c$ & $69.10 \pm 2.4 \mathrm{a}$ & $62.00 \pm 7.6^{c}$ & $64.00 \pm 2.6^{b}$ & $64.20 \pm 7.2^{b}$ \\
\hline Longitud de pierna & $37.50 \pm 8.0 \mathrm{a}$ & $31.10 \pm 1.9 b$ & $37.30 \pm 2.6$ a & $31.30 \pm 6.6 \mathrm{~b}$ & $38.10 \pm 4.9$ a & $36.50 \pm 9.4 \mathrm{a}$ \\
\hline Perímetro de grupa & $59.50 \pm 5.2^{b}$ & $57.00 \pm 9.1^{c}$ & $61.20 \pm 2.0 \mathrm{~b}$ & $66.90 \pm 6.5^{\mathrm{a}}$ & $64.70 \pm 3.4 \mathrm{a}$ & $64.70 \pm 6.6^{a}$ \\
\hline Ancho de grupa & $19.90 \pm 2.7$ & $21.70 \pm 2.4$ & $19.00 \pm 0.1$ & $21.40 \pm 2.6$ & $20.40 \pm 2.1$ & $21.10 \pm 2.2$ \\
\hline Ancho mayor de tórax & $22.50 \pm 2.8$ & $21.70 \pm 2.2$ & $22.10 \pm 2.4$ & $22.90 \pm 2.5$ & $23.70 \pm 2.6$ & $24.40 \pm 2.9$ \\
\hline Ancho menor de tórax & $18.30 \pm 0.4$ & $17.70 \pm 1.7$ & $16.00 \pm 1.5$ & $16.90 \pm 2.4$ & $18.10 \pm 2.3$ & $18.00 \pm 2.0$ \\
\hline Perímetro de tórax & $60.30 \pm 3.4^{c}$ & $60.70 \pm 2.4^{c}$ & $70.40 \pm 5.5$ a & $61.10 \pm 10.5^{c}$ & $65.30 \pm 8.4^{b}$ & $65.70 \pm 8.4^{b}$ \\
\hline PIT & $26.30 \pm 2.9$ & $23.00 \pm 2.1$ & $28.90 \pm 2.3$ & $24.80 \pm 4.0$ & $27.70 \pm 5.2$ & $26.50 \pm 4.8$ \\
\hline ICC & $0.31 \pm 0.1^{c}$ & $0.33 \pm 0.0 \mathrm{~b}$ & $0.25 \pm 0.2 d$ & $0.34 \pm 0.7^{b}$ & $0.36 \pm 0.5 \mathrm{a}$ & $0.36 \pm 0.0 \mathrm{a}$ \\
\hline
\end{tabular}

PIT= profundidad interna de tórax; ICC = Índice de compacidad de la canal [peso de la canal fría / longitud].

abcd Letras distintas en el mismo parámetro indican diferencia $(P<0.05)$.

superiores al $52 \%$ y las mayores áreas del ojo de chuleta(44,45,46), lo cual es ideal para satisfacer los requisitos más estrictos del mercado nacional e incluso del mercado internacional.

El índice de compacidad de la canal mostró algunas diferencias entre sexos y entre sistemas de producción, siendo mayor en el sistema intensivo; al respecto, se ha demostrado en otras investigaciones que conforme aumenta el tamaño de la canal se eleva el índice de compacidad y mejora la conformación ${ }^{(48,49)}$. La compacidad en la canal es un buen indicador del rendimiento comercial $(r=0.83$ $P<0.001)$, pues está altamente correlacionado con el peso de matanza $(r=0.98, P<0.001)$ y con el peso de la canal caliente $(r=0.91, \quad P<0.001)^{(50)}$. Asimismo, este índice se relaciona con la cantidad total de músculo de la canal, y entre mayor sea su valor, mejor es la aptitud cárnica en la canal, pues aumentan las medidas de anchura y espesor a costa de las de longitud( ${ }^{(8)}$.

\section{CONCLUSIONES E IMPLICACIONES}

Existe una amplia diversidad genética en la población ovina nacional, entre la que se encuentran razas puras y cruzamientos entre animales de lana y pelo en grado diverso. Se presentan fuertes diferencias en las características fenotípicas y la condición corporal de los ovinos (tipo, sexo, peso y edad al faenado) entre sistemas de producción y aún dentro de los mismos sistemas productivos. Hay una considerable variabilidad en las propiedades de la canal, presentando pesos, tamaños y morfologías totalmente diferentes, y con rangos muy amplios en todos sus atributos. Todo lo anterior limita fuertemente el mercado formal de la carne ovina, e implica la conveniencia de reordenar la producción nacional, dirigiéndola hacia una condición de calidad más uniforme, que satisfaga las exigencias actuales y futuras del mercado nacional, y esté en posibilidades de poder competir con el mercado internacional. Más aún si se quiere aprovechar la oportunidad de incursionar en nichos específicos de mercado.

\section{AGRADECIMIENTOS}

Se agradece al Fondo Sectorial de Investigación en Materia Agrícola, Pecuaria, Acuícola, Agrobiotecnológica y Recursos Fitogenéticos SAGARPA, CONACYT, COFUPRO por el financiamiento para la realización de este trabajo. Se agradece la colaboración de la MVZ Adriana Ríos Sánchez en la recolección y el análisis de muestras. 


\section{LITERATURA CITADA}

1. SAGARPA. Secretaría de Agricultura, Ganadería, Desarrollo Rural, Pesca y Alimentación. Estimación del Consumo Nacional Aparente 1990-2005. Carne de Ovino. Dirección General de Ganadería. http://www.sagarpa.gob.mx/ganaderia/Estadisticas/Lists/Estadstic as/Attachments/2/Estimaci\%C3\%B3n\%20del\%20Consumo\%20N acional\%20Aparente\%2019902005\%20Carne\%20de\%20ovino.pdf. Consultado Abr 8, 2016.

2. SIAP. Servicio de información agroalimentaria y pesquera. SAGARPA. Estacionalidad de carne en canal de ovino. Producción mensual 2005-2014. http://www.siap.gob.mx/opt/estacionalidad2/archivos/Carneovino. pdf. Consultado Abr 8, 2016.

3. SIAP. Servicio de información agroalimentaria y pesquera. SGARPA. Resumen nacional producción, precio, valor, animales sacrificados y precio. 2014. http://www.siap.gob.mx/resumen-nacionalpecuario/. Consultado Abr 8, 2016.

4. CONAPO. Consejo Nacional de Población. Datos de proyecciones. http://www.conapo.gob.mx/es/CONAPO/Proyecciones_Datos. Consultado Abr 8, 2016.

5. Boari R, Chuard N, Fernández V, Pouiller P. Mercado de ganados y carnes. Proyecciones 2023. OCDE-FAO 2014. http://www.agroindustria.gob.ar/site/ganaderia/bovinos/05=Merc ados/04=Carnes/_archivos/000003=Mercado\%20internacional\%2 0de\%20carnes/000001-

Proyecci\%C3\%B3n\%200CDE\%20FAO\%20carnes\%202014-

2023.pdf. Consultado May 25, 2016.

6. Wright DB, London K. First and second steps in statistics $2^{\text {nd }}$ ed. UK: Sage Publishing; 2009.

7. Boccard $\mathrm{R}$, Dumont $\mathrm{BL}$, Lefebre J. Étude de la production de la viande chez les ovins. La découpe des carcasses. Définition d'une découpe de référence. Anales de Zootechnie 1955;3:241-257.

8. Ruiz de Huidobro F, Miguel E, Cañeque V, Velasco S. Conformación, engrasamiento y sistemas de clasificación de la canal ovina. En: Cañeque V, Sañudo C editores. Estandarización de las metodologías para evaluar la calidad del producto (animal vivo, canal, carne y grasa) en los rumiantes. $1^{a}$ ed. España. Monografías INIA: Serie Ganadera No. 3. 2005:143-169.

9. Steel RGD, Torrie JH. Principles and procedures of statics: $A$ biometrical approach. $2^{\text {nd }}$. New York, USA: McGraw Hill Book Co; 1980.

10. [SAS] SAS/STAT User's Guide [release 9.1.3]. Cary NC, USA. SAS Inst. Inc. 2008.

11. Arteaga CJD. Mensaje institucional en el acto de inauguración. Memorias Foro Ovino del Estado de México. INIFAP. ICAMEX. 2012.

12. Symingoton RB. Studies on the adaptability of tree breeds of sheep to a tropical environment modified by altitude II. Response in body, skin and coat temperatures, cardio-respiratory frequencies and rate of moisture secretion of ewes to the diurnal fluctuation in temperature during the hottest of the year. J Agr Sci 1960;55(3):295-302.

DOI://dx.doi.org/10.1017/S0021859600023157.

13. Lara PSJ. Producción de ovinos de pelo en México: Materia genética para exportación. Congreso mundial de la lana y del cordero. Santiago de Querétaro, Qro. 2007:1-4.

14. Morteo GR, González GR, Torres HG, Nuncio OG, Becerril PC, Jaime $\mathrm{GS}$, et al. Efecto de la variación fenotípica en la resistencia de corderos Pelibuey a la infestación con nematodos gastrointestinales. Agrociencia 2004;38(5):395-404.

15. Figueroa CJA, Méndez MRD, Berruecos VJM, Gayosso VA, Ulloa AR, Acosta RR et al. Association between major histocompatibility complex microsatellites, fecal egg count, blood packed cell volume and blood eosinophilia in Pelibuey sheep infected with Haemoncus contortus. Vet Parasitol 2001;177(3-4):339-344.

16. De Lucas TJ. Estrategias reproductivas para aumentar la producción de corderos. 2011. http://spo.uno.org.mx/wpcontent/uploads/2011/07/9_jdlt_estrategias_repro.pdf. Consultado Ago 26, 2015.

17. Zavala ER, Ortiz OJ, Ramón UJP, Montalvo MP, Vásquez SA Sanginés GJR. Pubertad en hembras de cinco razas ovinas de pelo en condiciones de trópico seco. Zootec Trop 2008;26(4):465-473.

18. González GR, Salinas HRM, Garduza AG, Reyes MF. Componentes corporales en ovinos de pelo para abasto en el sureste mexicano. Zoot Trop 2014;32(1):23-32.

19. Partida PJA, Martínez RL. Composición corporal de corderos Pelibuey en función de la concentración energética de la dieta y del peso de sacrificio. Vet Mex 2010;41(3):177-190.

20. Peña F, Cano T, Domenech V, Alcalde MJ, Martos J, García MA et al. Influence of sex, slaughter weight and carcass weight on "noncarcass" and carcass quality in Segureña lambs. Small Ruminant Res 2005;60:247-254.

21. Yildirim A, Aksoy $Y$, Ocak N, Ulutaş Z. Some gastrointestinal tract characteristics of Karayaka ram lambs slaughtered at different weights. Scient World J 2014;1-6. http://dxdoi.org/10.1155/2014/379023.

22. Caro TW, Olivares EA, Araya AE. Relación entre peso de sacrificio y composición de la canal en corderos Suffolk. Agro Sur 1999;27(2):127-131.

23. Žgur S, Cividini A, Kompan D, Birtič D. The effect of live weight at slaughter and sex on lambs carcass traits and meat characteristics. Agr Conspec Sci 2003;3:155-159.

24. Fisher MW, Muir PD, Gregory NG. The animal welfare implications of depriving sheep of feed to facilitate transport and slaughter. MAF Technical Paper No. 2011/37. ISBN 978-0478-38407-9. New Zealand. 2011.

25. Carter LM, Gallo CB. Efectos del transporte prolongado por vía terrestre y cruces marítimos en transbordador sobre pérdidas de peso vivo y características de la canal en corderos. Arch Med Vet 2008;40:259-266.

26. Liste G, Miranda LGC, Campo MM, Villaroel M, María GA. Effect of lairage on lamb welfare and meat quality. Anim Prod Sci 2011;51(10):952-958.

27. Litherland AJ, Dynes RA, Moss EA. Factors affecting dressing-out percentage of lambs. Proc N Z Soc Anim Prod 2010;70:121-126.

28. Thompson JM, O'Halloran WJ, McNeill DMJ, Jackson-Hope NJ, May $\mathrm{TJ}$. The effect of fasting on live weight and carcass characteristics in lamb. Meat Sci 1987;20(4):293-309.

29. Mahgoub O, Lu CD. Effects of dietary energy density on fed intake, body weight gain and carcass chemical composition of Omani growing lambs. Small Ruminant Res 2000;37(1-2:35-42.).

30. Ríos RFG, Estrada AA, Plascencia A, López SMA, Castro PBI, Portillo $L J$ et $a l$. Influence of protein and energy in finishing diets for feed 


\section{CARACTERIZACIÓN DE LAS CANALES OVINAS PRODUCIDAS EN MÉXICO}

hair lambs: Growth performance, dietary energetics and carcass characteristics. Asian Aust J Anim Sci 2014;27(1):55-61.

31. Ahmad NA, Lloid HD. Effect of sex and dietary energy concentration on feed conversation ratio, growth and carcass characteristics in Merino x Border Leicester lambs. Proc Aust Soc Anim Prod 1986;16:119-122.

32. Abdel-baset N. Sayed. Effect of dietary energy levels on the performance and nutrient digestibility of lamb. Vet World 2009;2(11):418-420.

33. Partida PJA, Martínez RL, Braña VD. Desempeño productivo y propiedades de la canal en ovinos Pelibuey y sus cruzas con Suffolk o Dorset. Tec Pecu Mex 2009;47(3):313-322.

34. Cabrera NA, Rojas MP, Daniel RI, Serrano SA, López OM. Influencia de la suplementación sobre la ganancia de peso y calidad de la canal en borregos Dorper $\mathrm{X}$ Katahdin. Rev. UDO Agríc 2007;7(1):245-251.

35. Santos-Silva J, Mendes IA, Bessa RJB. The effect of genotype, feeding system and slaughter weight on the quality of light lambs. Livest Prod Sci 2002;76:17-25.

36. Fonti I Furnols $M$, Realine $C E$, Guerrero $L$, Peters $Z$, Nute GR, Sañudo $\mathrm{C}$ et al. Efecto del sistema de alimentación sobre la aceptabilidad de la carne ovina uruguaya en el mercado europeo. INIA-Uruguay Serie Técnica, 2007;168:107-112.

37. Garrido MD, Bañón S, Álvarez D. Medida del pH. En: Cañeque $\mathrm{V}$ Sañudo C editores. Estandarización de las metodologías para evaluar la calidad del producto (animal vivo, canal, carne y grasa) en los rumiantes. España. Monografías INIA: 2005; Serie ganadera No. 3.

38. Sañudos AC. Qualidade da carcaça e da carne ovina e caprina em face ao desenvolvimento da percepção do consumidor. Rev Bras Zootecn 2008;37(Supl Esp):143-160.

39. NMX-FF-106-SCFI-2006. Productos pecuarios. Clasificación de carne ovina en canal. Publicada en el Diario Oficial de la Federación el 4 de julio de 2006.

40. Moron-Fuenmayor OE, Clavero T. The effect of feeding system on carcass characteristics, non-carcass components and retail cut percentage of lambs. Small Ruminant Res 1999;34(1):57-64.
41. Ekiz B, Yilmaz A, Ozcan M, Kocak O. Effect of production system on carcass measurements and meat quality of Kivircik lambs. Meat Sci 2012;90:465-471.

42. Vnučec I, Dražaić V, Mioč B, Prpić Z, Pavić V, Antunović Z. Carcass traits and meat color of lamb from diverse production systems. Veterinarski Arhiv 2014;84:251-263.

43. Teke B, Ünal N. The effect of slaughter weight and sex on some slaughter traits of Akkarama and Morkarama and Turkis Merino lambs. Ankara Üniv Fak Der 2009;56:289-296.

44. Vázquez SET, Partida PJA, Rubio LMS, Méndez MRD. Comportamiento productivo y características de la canal en corderos provenientes de la cruza de ovejas Katahdin con machos de cuatro razas cárnicas especializadas. Rev Mex Cienc Pecu 2011;2(3):247-258.

45. Partida PJA, Casaya RTA, Rubio MS. Méndez MD. 2015. Effect of breed sire on carcass traits and meat quality of Katahdin lambs. J Food Res 2015;1(4):141-149.

46. López VMM, De la Cruz CL, Partida PJA, Torre HG, Becerril PC, Buendía RG, et al. Efecto de la raza paterna en las características de la canal de corderos en un sistema de producción comercial en Hidalgo, México. Rev Mex Cienc Pecu 2016;7(4):441-453.

47. De Souza JEL, De Sousa WH, Cavalcanti PE, Gonzaga NS, Queiroga $\mathrm{CF}$, Fontes $\mathrm{CM}$ et al. Effect of frame size on performance and carcass traits of Santa Ines lambs finished in a feedlot. Rev Bras Zootec 2013;42(4):284-290.

48. Sabbioni A, Beretti V, Zambini EM, Superchi P. Carcass and meat parameters in Cornigliese sheep breed as affected by sex and ageclass. Ital J Anim Sci 2016;15(1):2-9.

49. Teixeira A, Cadavez V, Delfa R, Bueno MS. Carcass conformation and joints composition by Suffolk and Merino precoce sire breeds. Span J Agric Res 2004;2(2):217-225.

50. Carrasco S, Ripoll G, Sanz A, Álvarez-Rodríguez J, Panea B, Joy M. Effect of feeding system on growth and carcass characteristics of Churra Tensina light lambs. Livest Sci 2006;121:56-63. 